\title{
CT perfusion imaging can detect residual lung tumor early after radiofrequency ablation: a preliminary animal study on both tumoral and peri-tumoral region assessment
}

\author{
Zenghui Cheng ${ }^{1,2 \#}$, Yixue Wang ${ }^{3 \#}$, Min Yuan ${ }^{1}$, Jianxiao Liang ${ }^{4}$, Yanling Feng ${ }^{5}$, Yuxin Shi ${ }^{1}$, Zhiyong Zhang ${ }^{1,6}$, \\ Fei Shan ${ }^{1}$
}

${ }^{1}$ Department of Radiology, Shanghai Public Health Clinical Center, Fudan University, Shanghai, China; ${ }^{2}$ Department of Radiology, Ruijin Hospital, Shanghai Jiaotong University, School of Medicine, Shanghai, China; ${ }^{3}$ Department of Critical Care Medicine, Children's Hospital of Fudan University, National Children's Medical Center, Shanghai, China; ${ }^{4}$ Department of Radiology, Dongying People's Hospital, Dongying, China; ${ }^{5}$ Department of Pathology, Shanghai Public Health Clinical Center, Fudan University, Shanghai, China; ${ }^{6}$ Department of Radiology, Zhongshan Hospital, Fudan University, Shanghai, China

Contributions: (I) Conception and design: Z Cheng, F Shan; (II) Administrative support: Z Zhang, Y Shi; (III) Provision of study materials: M Yuan, Y Feng; (IV) Collection and assembly of data: Z Cheng, Y Wang; (V) Data analysis and interpretation: Z Cheng, Y Wang, J Liang, F Shan; (VI) Manuscript writing: All authors; (VII) Final approval of manuscript: All authors.

\#These authors contributed equally to this work.

Correspondence to: Yuxin Shi, MD, PhD. Professor of Radiology, Shanghai Public Health Clinical Center, Fudan University, No. 2901 Caolang Road, Jinshan, Shanghai 201508, China. Email: shiyuxin@shphc.org.cn; Zhiyong Zhang, MD, PhD. Professor of Radiology, Zhongshan Hospital, Fudan University, No. 180 Fenglin Road, Xuhui, Shanghai 200032, China. Email: zhang.zhiyong@zs-hospital.sh.cn; Fei Shan, MD, PhD. Director of Radiology, Shanghai Public Health Clinical Center, Fudan University, No. 2901 Caolang Road, Jinshan, Shanghai 201508, China. Email: shanfei@shphc.org.cn.

Background: Radiofrequency ablation (RFA) is a minimally invasive procedure to treat lung cancer. Timely evaluation on residual lung tumor after RFA is crucial to the prognosis, hence, our objective is to assess CT perfusion (CTP) on detection of residual lung tumor early after RFA.

Methods: CTP imaging was performed in 24 lung VX2 tumor models 1 day before and within 1 hour after RFA. CTP maps with dual-input $(n=24)$ and single-input $[n=13$, with predominant ground glass opacity (GGO) after RFA] models were generated using the maximal slope method. Regions of interest were independently placed on the maximal cross-sectional tumor before and after RFA and on GGO after RFA by two thoracic radiologists. The bronchial flow (BF), pulmonary flow (PF) and perfusion index (PI) were compared between pre-RFA and post-RFA images. The parameters (BF, PF and PI of tumor; PF of GGO) of the complete and incomplete RFA groups were compared based on nicotinamide adenine dinucleotide hydrogen (NADH) and TdT-mediated dUTP nick-end labeling (TUNEL) staining and were correlated with the microvascular density (MVD).

Results: The BF and PF decreased after RFA (all P values <0.03). The decrease in $\mathrm{BF}$ and $\mathrm{PF}(\Delta \mathrm{BF}$ and $\triangle \mathrm{PF})$ in the complete RFA group was higher $(\mathrm{P}=0.01 ; 0.02)$. The areas under the curve (AUC) of $\triangle \mathrm{BF}$ and $\triangle \mathrm{PF}$ at 14.85 and $17.25 \mathrm{~mL} / \mathrm{min} / 100 \mathrm{~mL}$ in determination of tumor with complete ablation were 0.80 and 0.78 , respectively. $\triangle \mathrm{BF}$ was positively correlated with MVD $(\mathrm{P}=0.046, \mathrm{r}=0.468)$. PF of GGO with incomplete RFA was higher $(\mathrm{P}=0.001)$. The AUC of $\mathrm{PF} \leq 29.4 \mathrm{~mL} / \mathrm{min} / 100 \mathrm{~mL}$ in determination of tumor with complete ablation was 0.99.

Conclusions: CTP could detect residual lung tumor early after RFA in a rabbit model, which might provide a clinical solution to early treatment assessment after RFA.

Keywords: Radiofrequency ablation (RFA); CT perfusion (CTP); VX2 tumor; lung tumor

Submitted Jun 10, 2021. Accepted for publication Dec 06, 2021.

doi: $10.21037 /$ jtd-21-967

View this article at: https://dx.doi.org/10.21037/jtd-21-967

(C) Journal of Thoracic Disease. All rights reserved. 


\section{Introduction}

Radiofrequency ablation (RFA) has been adopted as a locally minimally invasive therapy for primary and oligometastatic lung tumors (especially, tumors less than $3 \mathrm{~cm}$ ) and is recognized as a safe and effective procedure (1-6). However, local tumor progression associated with incomplete ablation was reported to be $30-40 \%$ and $13-19 \%$ for primary and metastatic lung tumor, respectively (7). Hence, early detection of residual tumor after ablation is pivotal to the prognosis. The present method of evaluation after RFA is using conventional imaging (i.e., computed tomography and integrated with positron-emission tomography), which is occasionally imprecise due to complicated pathophysiological changes after ablation $(8,9)$. Functional imaging like CT perfusion (CTP) might have the potential to overcome this limitation.

CTP has been widely applied to the assessment of tumor angiogenesis, prediction of cancer pathologic grading and evaluation of tumor response to treatment (10). CTP evaluation of tumors after RFA has been mainly focused on liver cancer, which usually manifests as low and high blood perfusion within completely versus incompletely treated tumors (11-13). Few investigations of CTP on lung cancer after RFA have been carried out, and the majority utilized the single-input (usually systemic circulation) model of CTP without taking into account pulmonary arterial circulation (14). Lung cancers have been verified to have a good deal of pulmonary blood supply besides bronchial blood supply $(15,16)$. Thus, CTP with dual-input model on assessment of lung tumors after RFA might be more reasonable and accurate. Besides, previous investigations on treatment assessment using conventional imaging are usually based on the routine morphological pathology, which might underestimate the effect of RFA (17). The thermally fixed or apoptotic tumor cells sometimes manifest as normal vital cells on H\&E staining, yet the enzymes and proteins used to maintain cell activity have been denatured, and cells have been inactivated (17). Nevertheless, these pathological changes could be detected by specific staining, including nicotinamide adenine dinucleotide hydrogen (NADH) and TdT-mediated dUTP nick-end labeling (TUNEL) (18).

To achieve complete ablation of lung cancer, any peritumoral lung parenchyma within 5 to $10 \mathrm{~mm}$ needs to be ablated $(7,19,20)$. This area presents as necrosis, effusion and congestion from the inner zone to the outer zone on histopathology, accordingly (21), and manifests as ground glass opacity (GGO) on CT, which is the crucial area in the early assessment after RFA (8). Previous studies based on the morphological changes of unenhanced CT found that the size of GGO was associated with residual tumor and recurrence $(22,23)$. Hemodynamic changes of this region early after RFA could be assessed by pulmonary parenchymal perfusion based on single-input CTP, which would complement to the structural imaging assessment.

Therefore, we explored whether the use of dual-input together with single-input model of CTP based on NADH and TUNEL staining could accurately detect lung tumor viability both in tumoral and peri-tumoral regions early after RFA.

We present the following article in accordance with the ARRIVE reporting checklist (available at https://jtd. amegroups.com/article/view/10.21037/jtd-21-967/rc).

\section{Methods}

\section{Optimization of contrast material injection protocol for the dual-input model of computed tomography perfusion imaging}

We tested 4 different contrast material injection protocols (iohexol, $350 \mathrm{mg} / \mathrm{mL}$, injection rate and volume: $1.5 \mathrm{~mL} / \mathrm{s}$ $+1.0 \mathrm{~mL} ; 1.5 \mathrm{~mL} / \mathrm{s}+2.0 \mathrm{~mL} ; 2.0 \mathrm{~mL} / \mathrm{s}+1.0 \mathrm{~mL} ; 2.0 \mathrm{~mL} / \mathrm{s}$ $+2.0 \mathrm{~mL}$ ) (Table S1) among 40 New Zealand white rabbits, compared the parameters (Table S2) generated from the time-density curves (TDCs) of the pulmonary and bronchial arteries (Figure S1), and found that CTP with a contrast material injection rate of $1.5 \mathrm{~mL} / \mathrm{s}$ and volume of $2.0 \mathrm{~mL}$ was optimal for visualization of pulmonary and bronchial circulation of rabbit lung.

\section{Rabbit lung VX2 tumor modeling, RFA procedure and grouping}

This prospective study was under the approval of our center's Animal Care and Use Committee. Thirty New Zealand white rabbits (Shanghai Jiagan Biological Technology Co., Ltd.; permit number: SCXK2010-0028; 2.0 to $3.0 \mathrm{~kg}$ of weight, 3 to 5 months of age, with half males and half females) were implanted with VX2 tumor pieces (measuring $1 \mathrm{~mm}^{3}$, trimmed from the VX2 tumorbearing rabbits on their thighs, purchased from Shanghai Institutes for Biological Sciences). The tumor pieces were inoculated into either the right or left side of the lower lobe (distant from the heart, diaphragm, central vessels and 
main bronchi) percutaneously by a $17-\mathrm{G}$ coaxial needle $(6.8 \mathrm{~cm}$, Angiotech, Vancouver, BC, Canada) under CT guidance. The growth of implanted VX2 tumors was followed up by unenhanced CT at an interval of 1 week [according to the double time of lung VX2 tumor (24) and auricle VX2 tumor growth (25)], and the rabbits with VX2 tumors measuring 7 to $10 \mathrm{~mm}$ in the maximal diameter (two rabbits were randomly selected to test the blood supply to the implanted tumor by digital subtraction angiography and CT, and the tumoral angiogenesis was verified by $\mathrm{H} \& \mathrm{E}$ and CD31 staining) received a subsequent RFA procedure, which contained the following steps. First, 3\% pentobarbital sodium $(1 \mathrm{~mL} / \mathrm{kg}$, Dingguo Biotechnology Co., Ltd., Shanghai, China) was administered via the marginal ear vein to achieve general anesthesia. Second, a 17-G umbrella electrode (length of $10 \mathrm{~cm}, 10 \mathrm{sub}-$ needles, diameter of $2.0 \mathrm{~cm}$, Medsphere International Co., Ltd., Shanghai, China) or a 19-G single-needle electrode (length of $8 \mathrm{~cm}$, diameter of needle tip of $1 \mathrm{~cm}$, Medsphere International Co., Ltd., Shanghai, China) collocated with a radiofrequency generator (S-1500; Medsphere International Co., Ltd., Shanghai, China) was percutaneously placed into the implanted VX2 tumor under CT guidance. The subneedles of the umbrella electrode were opened $1.0 \mathrm{~cm}$ in diameter, and the single-needle electrode was inserted $1 \mathrm{~cm}$ into the tumor. Third, the ablation procedure either with a fixed power pattern of 25-30 W (umbrella electrode) or an initial power of $5 \mathrm{~W}$ with an increment of $5 \mathrm{~W}$ until the $25 \mathrm{~W}$ pattern (single-needle electrode) was set, and ablation was begun continuously until it ceased automatically due to the increasing impedance. This ablation procedure was carried out in a similar position once again on a 30 -second interval until the GGO measuring $5 \mathrm{~mm}$ appeared on the lung window of CT, suggesting a complete RFA (26).

The rabbits with implanted VX2 tumors, which served as the VX2 lung cancer models, were finally grouped after RFA into complete and incomplete RFA subgroups based on NADH and TUNEL staining.

\section{Computed tomography perfusion imaging}

The rabbits were fasted for 12 hours and anesthetized by administration of pentobarbital sodium $(3 \%, 1 \mathrm{~mL} / \mathrm{kg})$ into the marginal ear vein via a 24-gauge indwelling needle before CTP imaging. A supine position, with limbs and incisors fixed to a homemade examination pad and gauze bandages bound to the abdomen for controlling abdominal breathing, was utilized during examination. Additional sodium pentobarbital was administered for a stable respiratory rhythm at a rate under 20 per minute.

First, a positioning scan was carried out to determine the target tumor area. Then, a cine mode scan was performed after a 1-second delay of intravenous injection of $2 \mathrm{~mL}$ iohexol, followed by 4 or $5 \mathrm{~mL} 0.9 \%$ saline with the same speed of $1.5 \mathrm{~mL} / \mathrm{s}$ through a single indwelling needle (marginal ear vein) by a power injector (Stellant, SCT210, MEDRAD, Pittsburgh, USA). A 64-dector row CT scanner (Hitachi Scenaria 64, Hitachi Medical System, Kashiwa Shi, Chiba, Japan) was utilized to finish the whole scanning roughly from the thoracic inlet to the diaphragm with the tumor area as the center. The scan parameters were as follows: tube voltage, $100 \mathrm{kV}$; tube current, $80 \mathrm{~mA}$; $0.5 \mathrm{~s} /$ rotation; collimation, $64 \times 0.625$; matrix, $512 \times 512$; field of view (FOV), 90-120 mm; slice thickness/interval, $1.25 / 1.25 \mathrm{~mm}$; total acquisition time, $19 \mathrm{~s}(0.5 \mathrm{~s} /$ rotation, continuously repeated for 38 rotations); total dose-length product (DLP), $87.9 \mathrm{mGy} \cdot \mathrm{cm}$; window width (WW), $350 \mathrm{HU}$; and window level (WL), $40 \mathrm{HU}$.

\section{Inflated fixed lung with VX2 tumor, cross-sectional macro- and microscopic specimens}

The rabbits with VX2 tumor after RFA and CTP were sacrificed with an overdose of pentobarbital. The lungs were then immediately isolated, inflated and fixed using the Heitzman method. Those samples for NADH staining were inflated and fixed with optimal cutting temperature (OCT) compound $\left(37^{\circ} \mathrm{C}\right)$ after $10 \mathrm{~mL}$ air inflation until they appeared not collapsed.

The inflated and fixed lungs were set at the supine position and scanned with a slice thickness of $2.5 \mathrm{~mm}$ to position the central slice of the VX2 tumor after RFA under circumstances of the lung window. The cross-sectional macrospecimens with the same thickness were then sliced under guidance of the laser positioning line from the central slice to both ends and fixed in formalin overnight for paraffin embedding of the histological specimen $(5 \mu \mathrm{m})$ and staining (H\&E, CD34 and TUNEL) or were immediately frozen for NADH staining.

\section{$H \& E, C D 31, N A D H$ and TUNEL staining and evaluation}

The sections with $5 \mu \mathrm{m}$ thickness were first stained with 
hematoxylin-eosin for routine morphological assessments and were then reacted with a $3 \% \mathrm{H}_{2} \mathrm{O}_{2}$-methanol solution for 12 minutes, incubated in $100 \mu \mathrm{L}$ serum for 15 minutes and with the CD31 antibody (primary and secondary antibodies, Arigo Biolaboratories Corp., Taiwan, China) within 1 day at room temperature for microvessel density (MVD) evaluation according to the Weidner methods (27), which were detailed as follows. First, one single microvessel was defined as one isolated CD31 positively stained vascular endothelial cell or clusters of CD31 positively stained cells with or without cavity formation and a branching structure with a clear gap. The diameter of the cavity was larger than 8 red blood cells, and those with a muscular layer and CD31 positively stained cells in the necrosis and peri-tumoral areas were not calculated as one single microvessel. Second, the "hot-spot" area was defined as a strong staining area on 40-fold magnified views. Third, five views were randomly selected on the 200-fold magnified of the "hot-spot" area, and the average was defined as the MVD.

Air- and OCT-compound-fixed fresh lung with VX2 after RFA within 10 minutes was frozen at $-80{ }^{\circ} \mathrm{C}$ for 2 to 3 minutes and cut into slices with a thickness of $10 \mu \mathrm{m}$. Then, the sections were incubated in $10 \mathrm{~mL}$ TrisHCL buffer ( $\mathrm{pH}=7.4)$ containing $8 \mathrm{mg} \mathrm{NADH}$ (Beyotime Biotechnology, Shanghai, China) and $10 \mathrm{mg}$ NBT (Beyotime Biotechnology, Shanghai, China) at $37^{\circ} \mathrm{C}$ for 30 minutes (28). The positive NADH staining was dark blue, indicating that those cells were viable, while the negative staining was colorless, indicating that those cells died as a result of $\mathrm{NADH}$ diaphorase inactivation immediately after cell death $(29,30)$.

The TUNEL staining procedure was performed on the paraffin embedded sections according to the modified Roche protocol (Version 11, April 2006) using the Roche cat. for in situ cell death detection kit (No. 11684795910). Positive staining of massive DNA fragments caused by coagulative necrosis in the center of ablation was classified as a true positive result instead of a false positive in the study. TUNEL-positive staining was defined as nuclei with green light under UV excitation indicating cell apoptosis or death, whereas negative staining was defined as nuclei with blue light without excitation indicating cell viability.

All the above stained histological sections were first scanned by a digital panoramic imaging system (Pannoramic-250, 3D HISTECH Ltd., Budapest, Hungary) with 200-fold magnification and then reviewed and analyzed by a pathologist with more than 30 years of experience in diagnostic pathology and various staining techniques.

\section{Data processing and analysis}

All CTP images (DICOM format) were transferred to the Vitrea station (Vitrea FX, version 6.5.3, Minnetonka, $\mathrm{MN}$, USA).

The dual-input perfusion model was utilized in the body perfusion packs for the assessment of the VX2 tumor before and after RFA. First, non-rigid correction was performed for the respiratory movement. Second, the region of interest (ROI) of the pulmonary artery trunk, descending aorta and left atrium was delineated on the transverse image at the mediastinal window. Third, the according TDC was generated automatically and adjusted to show the left atrium enhancement peak point in the intersection of the TDC of the pulmonary artery truck and descending aorta (cutpoint of the pulmonary and systemic circulation). Fourth, the dual-input CTP pseudo-color mapping (displaying calculated parameters in rainbow colors) was calculated with the maximum slope method $(31,32)$ at $C T$ values ranging from -80 to $150 \mathrm{HU}$. Finally, ROIs of the VX2 tumor before and after RFA (referring to the images before RFA) were delineated on the maximal axial images while avoiding the lobar bronchi and vessels to generate the perfusion parameters of bronchial flow (BF, unit: $\mathrm{mL} / \mathrm{min} / 100 \mathrm{~mL}$ ), pulmonary flow (PF, unit: $\mathrm{mL} / \mathrm{min} / 100 \mathrm{~mL}$ ) and lung perfusion index (PI, unit: \%).

The single-input perfusion model was utilized in the lung perfusion packs for the assessment of the peritumoral "safe zone". First, non-rigid correction was performed for the respiratory movement. Second, the ROI of the pulmonary artery truck and lung parenchyma of the right lower lobe (avoiding the area adjacent to the heart and diaphragm) was delineated on the transverse image at the mediastinal and lung window. Third, the TDC was generated automatically and then adjusted by verifying each ROI in a similar region at different time points. Fourth, the single-input CTP pseudo-color mapping was calculated with the maximum slope method at a CT value ranging from -400 to $-50 \mathrm{HU}$. Finally, the ROIs of the GGO after RFA were delineated from the central to peripheral ablation zone based on different contours caused by the decreasing temperature of the lung tissue on the pseudo-color map; accordingly, the PF was generated.

The following steps were used to well register between CTP images and pathological images: firstly, cross-sectional CTP images across the maximal axis of the tumor before RFA were marked by both tumor contour and adjacent anatomic structures (e.g., tracheal carina, ascending aorta, 
heart atrium and ventricle); secondly, cross-sectional CTP images across the maximal axis of the tumor after RFA were identified by cross checking with the marked CTP images in the prior step; thirdly, large histological section with H\&E staining which were well correlated with CTP images were identified by cross-referring to both tumor contour and adjacent lung parenchymal structures (e.g., lobar and segmental bronchus, pulmonary artery and vein). NADH and TUNEL staining histological sections were then made for further subgrouping.

The measurement was blindly performed by two radiologists ( $\mathrm{ZC}$ and $\mathrm{FS}$, with 10 and 15 years of experience in thoracic diagnostic imaging, respectively), and the mean value was used.

\section{Statistical analysis}

The values of PI, BF, PF in the complete and incomplete groups before and after RFA were compared by either a two-tailed unpaired $t$-test (data in Gaussian distribution tested by the Kolmogorov-Smirnov test) or a two-tailed Mann-Whitney $U$ test (data in non-Gaussian distribution). The receiver operating characteristic (ROC) curve was used to test the differentiating ability of the perfusion parameter in terms of area under the curve (AUC), sensitivity, specificity and positive likelihood ratio $(\mathrm{LR}+)$. The correlation between the significant perfusion parameter and MVD was tested by either a one-tailed Pearson correlation (data in Gaussian distribution) or Spearman correlation (data in non-Gaussian distribution). The coefficients of correlation above and below 0 indicated positive and negative correlations, respectively. An absolute value above 0.6 indicated high correlation, a value between 0.4 and 0.6 indicated correlation, and a value below 0.4 indicated no correlation. All the statistics were carried out by STATA (Version 14.1.408, Statacorp, TX, USA), and the statistical charts were created by GraphPad Prism (Version 8.0.0, GraphPad Software, CA, USA). All significant levels were set at 0.05 .

\section{Ethical statement}

Experiments were performed under a project license (No. 2017-A015-01) granted by the institutional ethics committee board of Shanghai Public Health Clinical Center, in compliance with Shanghai Public Health Clinical Center's institutional guidelines for the care and use of animals. A protocol was prepared before the study without registration.

\section{Results}

\section{Rabbit lung VX2 tumor model with RFA and CTP}

There were 28/30 successfully implanted lung VX2 tumor models. Of these 28 models, 2 were sacrificed to test for tumoral angiogenesis, and another 2 died from hemopneumothorax and bronchopleural fistula during the RFA procedure. The remaining 24 models successfully received both dual-input CTP and RFA. Of the 24 finally enrolled models, 11/24 models had no prominent GGO adjacent to the tumor after RFA, which might be caused by subsequent lung parenchymal collapse, congestion, or consolidation. These 11/24 models were not further investigated by single-input CTP on account of avoiding the potential influence on the blood supply of this peri-tumor region. Consequently, 13/24 models with prominent GGO were finally enrolled to investigate the single-input CTP.

\section{Dual-input CTP changes in rabbit lung VX2 tumor early after RFA}

The mean value of $\mathrm{BF}$ and the median value of $\mathrm{PF}$ decreased $14.10 \pm 15.09 \mathrm{~mL} / \mathrm{min} / 100 \mathrm{~mL}$ and $15.60(6.00-26.00)$ $\mathrm{mL} / \mathrm{min} / 100 \mathrm{~mL}$ after RFA $(\mathrm{P}=0.0001 ;<0.0001, \alpha=0.05)$, respectively, and the corresponding value of the complete RFA group decreased more than that of the incomplete RFA group $(\mathrm{P}=0.01 ; 0.02, \alpha=0.05)$. The mean value of PI slightly decreased $0.01 \pm 0.15$ after RFA; however, there was no significant difference $(\mathrm{P}=0.7058, \alpha=0.05)$. The mean value of PI in the complete RFA group slightly increased early after RFA, while the mean value of PI in the incomplete RFA group slightly decreased early after RFA, yet there were no significant differences $(\mathrm{P}=0.23, \alpha=0.05)$ (Tables 1,2; Figures 1,2). The ROC curve shows that using a decrease of $\Delta \mathrm{BF} \geq 14.85 \mathrm{~mL} / \mathrm{min} / 100 \mathrm{~mL}$ and $\Delta \mathrm{PF} \geq 17.25 \mathrm{~mL} / \mathrm{min} /$ $100 \mathrm{~mL}$ as a threshold to determine complete RFA of lung VX2 tumors could produce AUC of 0.80 and 0.78 , sensitivity of $75.00 \%$ and $75.00 \%$, specificity of $66.67 \%$ and $66.67 \%$, and LR+ of 2.25 and 2.25 , respectively.

\section{Changes in single-input CTP of the peritumoral "safe zone" of rabbit lung VX2 tumor after RFA and its role in early evaluation}

There were 13 rabbit lung VX2 tumors with predominant 
Table 1 Dual-input CT perfusion parameter changes in the implanted VX2 tumor after RFA

\begin{tabular}{lccc}
\hline Parameters & Pre-RFA $(\mathrm{N}=24)$ & Post-RFA $(\mathrm{N}=24)$ & $\mathrm{P}$ \\
\hline $\mathrm{BF}(\mathrm{mL} / \mathrm{min} / 100 \mathrm{~mL})$ & $25.50 \pm 14.95$ & $11.40 \pm 5.77$ & $0.0001^{*}$ \\
$\mathrm{PF}(\mathrm{mL} / \mathrm{min} / 100 \mathrm{~mL})$ & $25.40(11.45-35.43)$ & $11.40(6.58-15.33)$ & $<0.0001^{*}$ \\
$\mathrm{PI}$ & $0.58 \pm 0.12$ & $0.57 \pm 0.13$ & 0.7058 \\
\hline
\end{tabular}

Value of $\mathrm{BF} / \mathrm{PI}$ : mean $\pm \mathrm{SD}$; value of PF: median $(95 \% \mathrm{Cl}) .{ }^{*}, \mathrm{P}$ values indicate significant difference. RFA, radiofrequency ablation; BF, bronchial flow; PF, pulmonary flow; PI, perfusion index.

Table 2 Dual-input CT perfusion changes in the implanted VX2 tumor early after RFA in the complete and incomplete RFA subgroups

\begin{tabular}{lccc}
\hline Parameters & Complete RFA $(\mathrm{N}=12)$ & Incomplete RFA (N=12) & P \\
\hline$\Delta \mathrm{BF}(\mathrm{mL} / \mathrm{min} / 100 \mathrm{~mL})$ & $21.66 \pm 14.61$ & $6.54 \pm 11.76$ & $0.01^{*}$ \\
$\Delta \mathrm{PF}(\mathrm{mL} / \mathrm{min} / 100 \mathrm{~mL})$ & $22.90(14.35-34.38)$ & $9.90(1.68-19.03)$ & $0.02^{*}$ \\
$\Delta \mathrm{PI}$ & $0.03 \pm 0.15$ & $0.05 \pm 0.15$ & 0.23
\end{tabular}

$\Delta=\mid$ post-RFA - pre-RFA|; value of $\triangle \mathrm{BF} / \triangle \mathrm{PI}$ : mean $\pm \mathrm{SD}$; value of $\triangle \mathrm{PF}$ : median $(95 \% \mathrm{Cl}) .{ }^{*}$, $\mathrm{P}$ values indicate significant difference. $\mathrm{RFA}$, radiofrequency ablation; $\mathrm{BF}$, bronchial flow; $\mathrm{PF}$, pulmonary flow; $\mathrm{PI}$, perfusion index.

GGO immediately after RFA, and 6 of them were classified as the incomplete RFA group according to $\mathrm{H} \& \mathrm{E}$ and NADH staining according to the vital tumor area shown in the peri-tumor region. The mean PF value in the corresponding GGO was $39.07 \mathrm{~mL} / \mathrm{min} / 100 \mathrm{~mL}$. The remaining 7 models were classified as the complete RFA group, and the mean value of $\mathrm{PF}$ in the GGO was $18.83 \mathrm{~mL} / \mathrm{min} / 100 \mathrm{~mL}$. As a control, the mean PF of GGO of the normal lung after RFA was $20.66 \mathrm{~mL} / \mathrm{min} / 100 \mathrm{~mL}$. There were significant differences among these three subgroups ( $\mathrm{P}=0.0009, \alpha=0.05 / 3)$. The mean $\mathrm{PF}$ of $\mathrm{GGO}$ in the incomplete RFA subgroup was larger than that in the complete RFA subgroup $(\mathrm{P}=0.0012, \alpha=0.05 / 3)$ and in the normal lung control subgroup $(\mathrm{P}=0.0087, \alpha=0.05 / 3)$, while there was no significant difference between the latter two subgroups ( $\mathrm{P}=0.8447, \alpha=0.05 / 3)$ (Table 3; Figure 3). The ROC test showed that using $\mathrm{PF} \geq 29.4 \mathrm{~mL} / \mathrm{min} / 100 \mathrm{~mL}$ as a threshold to determine incomplete RFA of GGO could produce an AUC of 0.99 , sensitivity of $100 \%$, specificity of $91.67 \%$, and LR+ of 12.00 .

\section{Correlations between CTP parameters and MVD}

$\triangle \mathrm{BF}$ positively correlated with MVD $(\mathrm{P}=0.0456, \mathrm{r}=0.4684$, $\alpha=0.05$, Pearson's correlation), while there was no significant correlation among $\triangle \mathrm{PF}, \triangle \mathrm{PI}$ and $\mathrm{MVD}(\mathrm{P}=0.1437$, $r=0.3059, \alpha=0.05 ; P=0.1000, r=-0.3645, \alpha=0.05$, Pearson's correlation).

\section{Discussion}

To date, CTP based on a single-input model has been investigated for the evaluation of various treatments of lung cancer, including chemotherapy, targeted therapy, radiotherapy and interventional therapy. The majority of these studies confirmed CTP as a useful method and found that BF and permeability surface (PS) product decreased after treatment (33-37). Few studies have been carried out on thermal ablation, and the results have varied. A study found that BF and PS decreased and correlated well with the results assessed by the response evaluation criteria in solid tumors (RECIST) (38), while another study on microwave ablation did not find a difference (39). The reason might be that the algorithm being chosen is a single-input model, and there could be some pulmonary blood supply to the tumor and transiently increased pulmonary blood perfusion around the ablation at an early stage. DI-CTP might have the potential to solve this problem, as was found by our study that $\mathrm{BF}$ and $\mathrm{PF}$ decreased at an early stage after RFA. The possible mechanism might be that thermal damage during ablation directly or indirectly destroys the microvessels of lung tumors, triggering a large number of round cells in the interstitium infiltrating the capillary wall and causing degenerative changes, which eventually lead to occlusion and necrosis of tumor blood supply vessels (40) and a prominent decrease in microcirculatory perfusion. In addition, we found that both $\mathrm{BF}$ and $\mathrm{PF}$ decreased more in the complete ablation groups than in the incomplete group, 

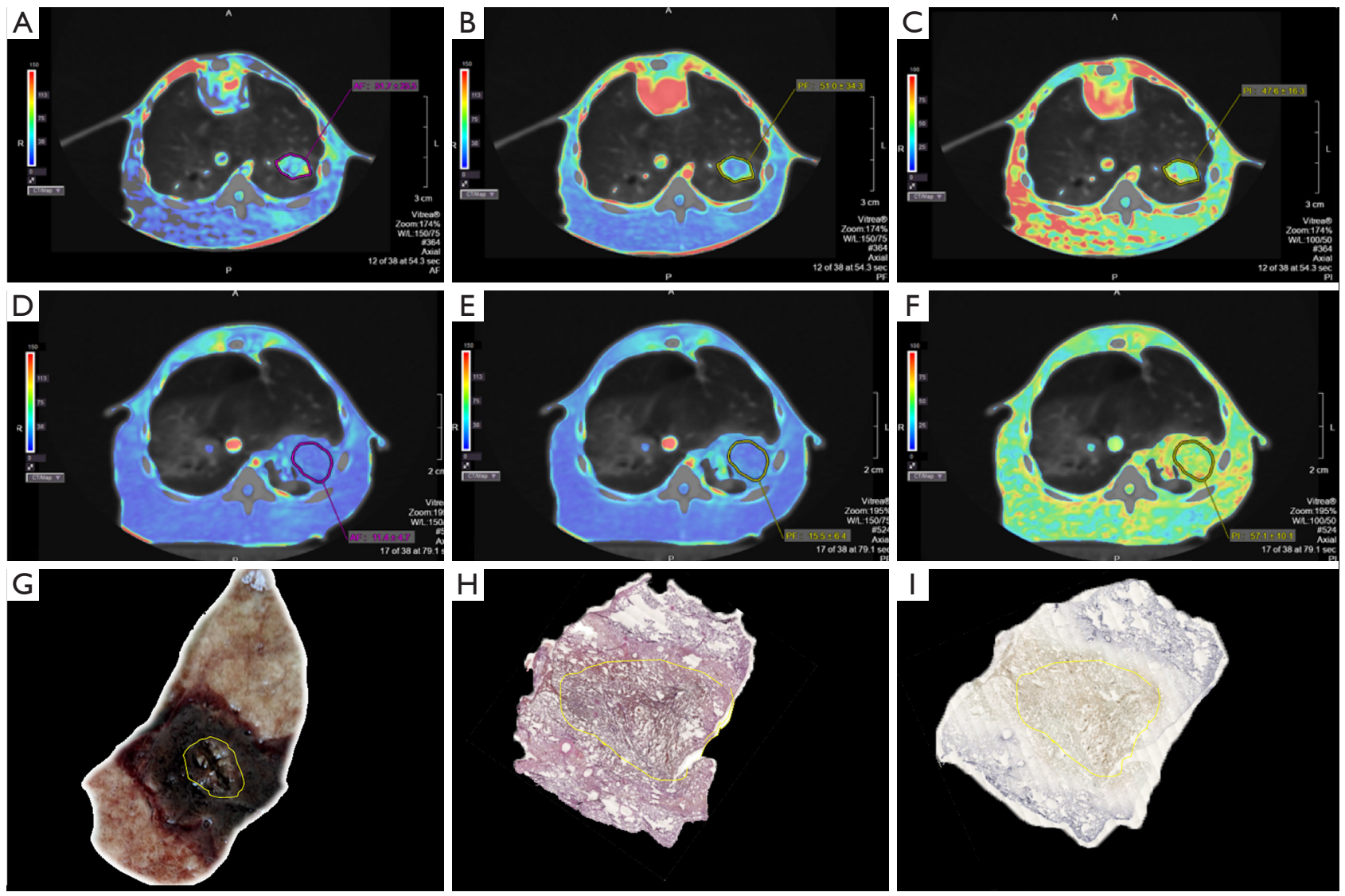

Figure 1 Dual-input CT perfusion changes in the implanted lung VX2 tumor with complete ablation based on H\&E and NADH staining within 1 hour after RFA. (A-C) BF, PF, PI map of the VX2 tumor pre-RFA; (D-F) BF, PF, PI map of the VX2 tumor within 1 hour post-RFA; corresponding value of ROI: $51.7 \mathrm{~mL} / \mathrm{min} / 100 \mathrm{~mL}, 51 \mathrm{~mL} / \mathrm{min} / 100 \mathrm{~mL}, 47.6 \% ; 11.4 \mathrm{~mL} / \mathrm{min} / 100 \mathrm{~mL}, 15.5 \mathrm{~mL} / \mathrm{min} / 100 \mathrm{~mL}, 57.1 \%$. (G-I) Macropathology of cross-sectional lung specimens, H\&E staining (1×), and NADH staining (1×). ROI with yellow color illustrating the VX2 tumor post-RFA; dense and hematoxylin-stained tumor cells are visible, while negative staining on NADH indicates nonviable tumor cells. NADH, nicotinamide adenine dinucleotide hydrogen; RFA, radiofrequency ablation; BF, bronchial flow; PF, pulmonary flow; PI, perfusion index; ROI, region of interest.

which provides a basis for the early evaluation of RFA. The PI was reported to be a good perfusion biomarker in differentiation of malignant from benign solitary pulmonary nodules with an AUC of 0.92 , and the PI of malignancy was smaller than that of benignity (41). Similar to this investigation, we found a trend in which PI increased in the complete RFA group but decreased in the incomplete RFA group, although there was no statistically significant difference, which could be explained by changes in the dualinput blood supply of the tumor after RFA. BF decreased more than $\mathrm{PF}$ in the complete RFA group; however, $\mathrm{BF}$ decreased slightly more than $\mathrm{PF}$ in the incomplete RFA group. The reason for the non-statistically significant difference might be the miscellaneous effects of the PI, for instance, tumor size and volume: the larger the volume was, the smaller the PI was (42). The variance in volume of the VX2 tumor in the study might be one factor accounting for this observation. In addition, the implanted tumor was mainly peripherally located, which might be another factor, as was reported that the proportion of pulmonary blood supply in peripheral lung cancer is larger than that in centrally located lung cancer (14).

The GGO appearance around the VX2 tumor after RFA is the key area to be carefully assessed, which is pathophysiologically complicated. The GGO could be vasculogenic as a result of reactive increasement in microvascular blood volume after lung tissue damage or non-vasculogenic due to bronchoalveolar destruction, 

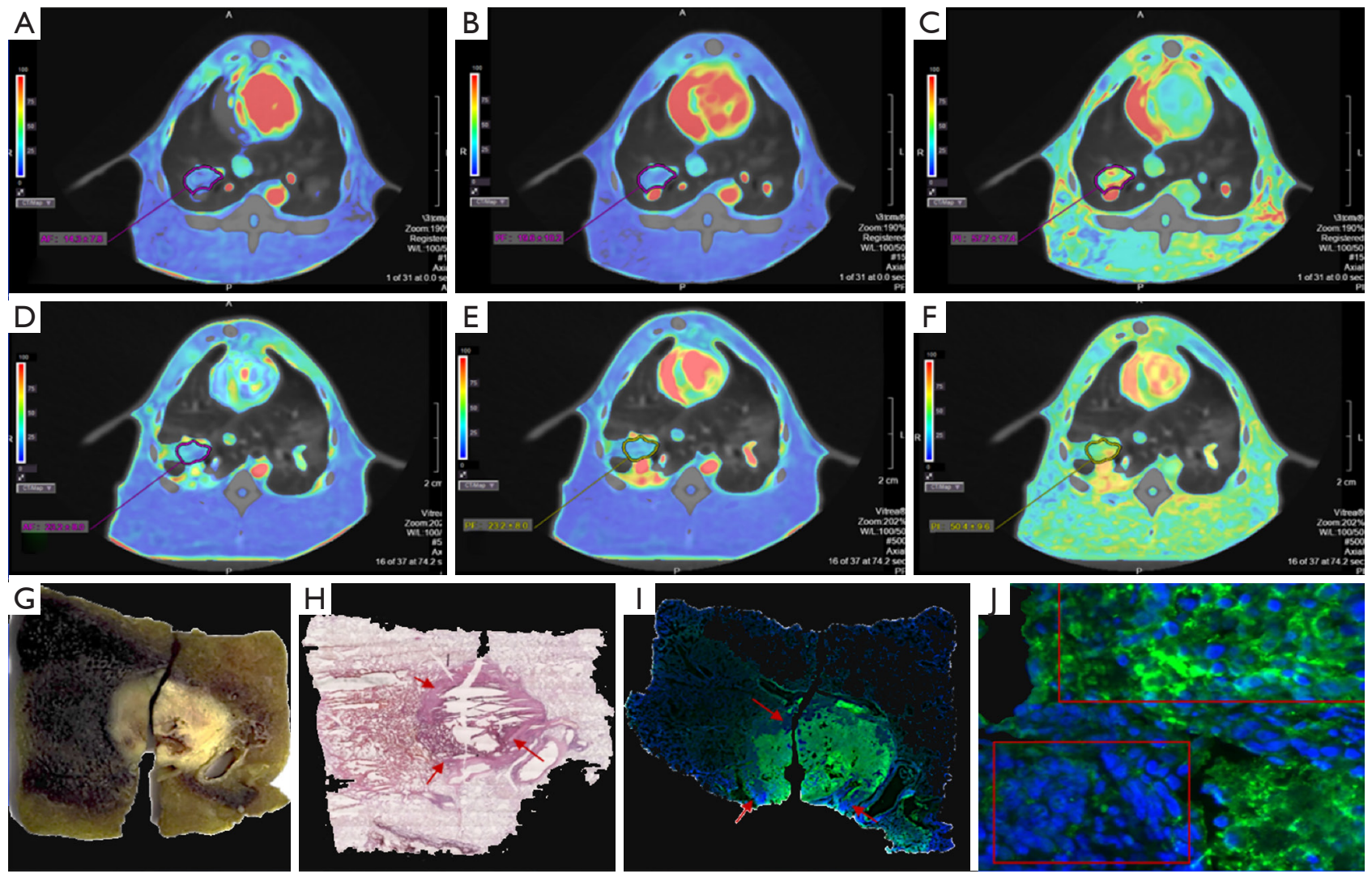

Figure 2 Dual-input CT perfusion changes in the implanted lung VX2 tumor with incomplete ablation based on H\&E and TUNEL staining within 1 hour after RFA. (A-C) BF, PF, PI map of the VX2 tumor pre-RFA; (D-F) BF, PF, PI map of the VX2 tumor within 1 hour post-RFA; corresponding value of ROI: $14.3 \mathrm{~mL} / \mathrm{min} / 100 \mathrm{~mL}, 19.6 \mathrm{~mL} / \mathrm{min} / 100 \mathrm{~mL}, 57.7 \% ; 23.2 \mathrm{~mL} / \mathrm{min} / 100 \mathrm{~mL}, 23.2 \mathrm{~mL} / \mathrm{min} / 100 \mathrm{~mL}$, $50.4 \%$. (G-J) macropathology of cross-sectional lung specimens, H\&E staining (1×), TUNEL staining (1×), and TUNEL staining (200×). Area with red arrows illustrating vital part of the VX2 tumor, blue-stained on TUNEL staining; left lower red rectangle indicates vital tumor cells; right upper red rectangle indicates almost nonviable tumor cells. TUNEL, TdT-mediated dUTP nick-end labeling; RFA, radiofrequency ablation; BF, bronchial flow; PF, pulmonary flow; PI, perfusion index; ROI, region of interest.

Table 3 Single-input CT perfusion changes in the peri-tumoral "safe zone" early after RFA in different subgroups

\begin{tabular}{lcc}
\hline Subgroups & $\mathrm{PF}(\mathrm{mL} / \mathrm{min} / 100 \mathrm{~mL}$, mean $\pm \mathrm{SD})$ & $\mathrm{P}$ \\
\hline Complete RFA $(\mathrm{N}=7)$ & $18.83 \pm 4.36$ & $0.0012^{\circledR}$ \\
Incomplete RFA $(\mathrm{N}=6)$ & $39.07 \pm 6.98$ & $0.0087^{\#}$ \\
Normal lung control $(\mathrm{N}=5)$ & $20.66 \pm 8.30$ & $0.8447^{\&}$ \\
$\mathrm{P}$ & $0.0009^{\star}$ & \\
\hline
\end{tabular}

*, P value of comparison between the 3 groups; ${ }^{\circledR}$, P value of comparison between complete and incomplete RFA; ${ }^{\#}, \mathrm{P}$ value of comparison between incomplete RFA and normal control; ${ }^{\&}$, P value of comparison between complete RFA and normal control; numbers in italic denote significant differences. RFA, radiofrequency ablation; PF, pulmonary flow.

collapse or some tumor cell infiltration in a lepidic pattern through the alveolar pore (43). The non-vasculogenic GGO can be easily assessed by CTP with the decreased PF after
RFA; however, the vasculogenic GGO can be discerned by the increased vessel diameter within the GGO (44) or dualenergy CTP when the diameter of the corresponding vessel 

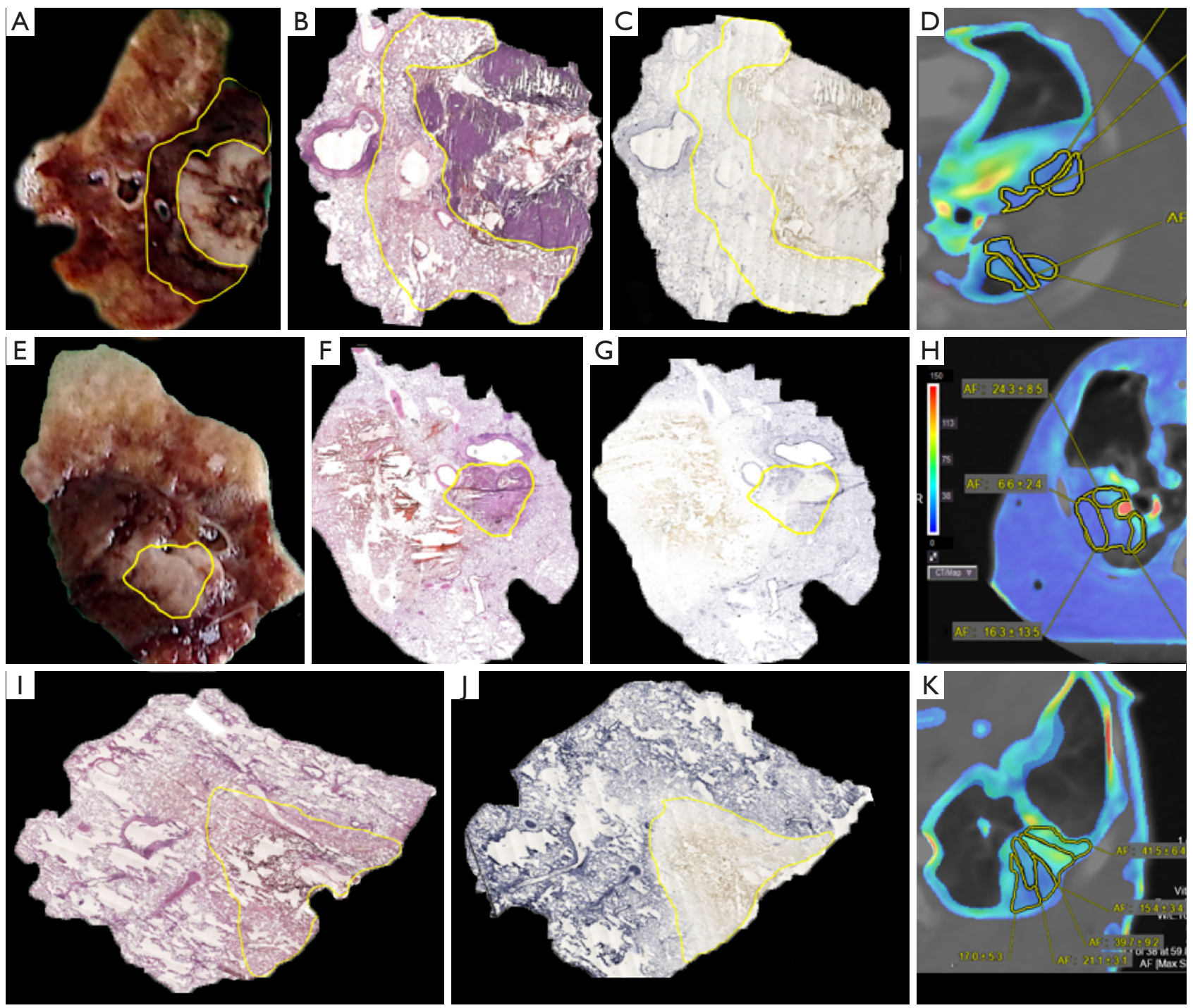

Figure 3 Single-input CT perfusion changes in the "safe zone" around implanted lung VX2 tumor and normal lung within 1 hour after RFA based on $\mathrm{H} \& \mathrm{E}$ and $\mathrm{NADH}$ staining. (A-D) Cross-sectional lung specimen (A), H\&E staining (B), NADH staining (C) and pulmonary parenchymal CTP (D) of GGO around a complete lung VX2 tumor after RFA. The ROI in yellow indicates devitalized tissues (on NADH staining); mean PF of the corresponding ROI: $12.75 \mathrm{~mL} / \mathrm{min} / 100 \mathrm{~mL}$. (E-H) Cross-sectional lung specimen (E), H\&E staining (F), NADH staining (G) and pulmonary parenchymal CTP $(\mathrm{H})$ of GGO around an incomplete lung VX2 tumor after RFA. The ROI in yellow indicates vital tissues (blue part on NADH staining-vital); PF of corresponding ROI: $34.9 \mathrm{~mL} / \mathrm{min} / 100 \mathrm{~mL}$. (I-K) H\&E staining (I), B-NADH staining (J) and pulmonary parenchymal CTP (K) of GGO of normal lung after RFA. The ROI in yellow indicates no vital tissue (on NADH staining); the mean PF of the left lower part of ROI: $12.75 \mathrm{~mL} / \mathrm{min} / 100 \mathrm{~mL}$; the right upper part of ROI indicating congestion. RFA, radiofrequency ablation; NADH, nicotinamide adenine dinucleotide hydrogen; CTP, CT perfusion; GGO, ground glass opacity; ROI, region of interest; PF, pulmonary flow.

cannot be verified (43). Previous studies usually uses a size of at least $5 \mathrm{~mm}$ and integrity of GGO as the standard for complete ablation (45); however, predicting success based only on the morphology is not precise. In this study, we used the CTP with a calculated threshold of the CT value between -400 and $-50 \mathrm{HU}$ to assess the lung pulmonary parenchyma with GGO and found that the PF of the residual tumor area was larger than that of the complete ablation area with either a tumor or normal lung, which is similar to the report on the instant evaluation of normal 
porcine liver with CTP (46), suggesting that CTP could be a promising method in the early evaluation of GGO around lung tumors after RFA.

Regarding the correlation between perfusion parameters and MVD, we found that only $\triangle \mathrm{BF}$ positively correlated with MVD, which was consistent with the results from studies based on the single-input model CTP (47-49). The reason for the lack of correlation between the other two parameters and MVD might be due to the inability to differentiate arterioles from venules using this counting method, and there was still some overlap between the bronchial and pulmonary blood supply, even with the maximum slope method of dual-input CTP.

Admittedly, there were several limitations in this study. First, the artifacts caused by respiratory movements might affect the perfusion parameters; however, a non-rigid movement correction was utilized to alleviate this effect. Second, delineation of the VX2 tumor on the maximal crosssection might not fully reflect the perfusion changes of the whole tumor (50) because there was spatial heterogeneity in the tumoral blood supply (51). A delineation of the whole tumor would be warranted to verify the present results. Third, there were no comparisons between different mathematical algorithms on the calculation of perfusion parameters, as there might be differences in the treatment evaluation based on perfusion (52). However, a study on the CTP calculated with different mathematical algorithms of normal porcine liver after RFA found that all perfusion parameters could discriminate vital from necrotic tissues (46). Last, neither inter- nor intra-observer consistency tests were carried out; nevertheless, the intra-observer consistency was stable on volume perfusion CT, and the inter-observer consistency was ameliorated after training (53).

In conclusion, CTP with a dual-input model and a singleinput model could evaluate the lung VX2 tumor and the "safe zone" after RFA, respectively, at an early stage. The combined method could be used for comprehensively early evaluation and thus could provide clues in clinical practice.

\section{Acknowledgments}

We sincerely acknowledge Cheng Ren (M.A., Hangzhou Yasheng Brand Design Co., Ltd.) for her assistance in figure editing. We also thank all the laboratory rabbits in this study. Funding: This work was supported by the National Natural Science Foundation of China (grant number 81301223) and the Clinical Research Plan of SHDC (grant number SHDC2020CR3080B).

\section{Footnote}

Reporting Checklist: The authors have completed the ARRIVE reporting checklist. Available at https://jtd. amegroups.com/article/view/10.21037/jtd-21-967/rc

Data Sharing Statement: Available at https://jtd.amegroups. com/article/view/10.21037/jtd-21-967/dss

Conflicts of Interest: All authors have completed the ICMJE uniform disclosure form (available at https://jtd.amegroups. com/article/view/10.21037/jtd-21-967/coif). All authors report that the study is funded by National Natural Science Foundation of China (grant number 81301223) and the Clinical Research Plan of SHDC (grant number SHDC2020CR3080B). The authors have no other conflicts of interest to declare.

Ethical Statement: The authors are accountable for all aspects of the work in ensuring that questions related to the accuracy or integrity of any part of the work are appropriately investigated and resolved. Experiments were performed under a project license (No. 2017-A015-01) granted by institutional ethics committee board of Shanghai Public Health Clinical Center, in compliance with Shanghai Public Health Clinical Center's institutional guidelines for the care and use of animals. A protocol was prepared before the study without registration.

Open Access Statement: This is an Open Access article distributed in accordance with the Creative Commons Attribution-NonCommercial-NoDerivs 4.0 International License (CC BY-NC-ND 4.0), which permits the noncommercial replication and distribution of the article with the strict proviso that no changes or edits are made and the original work is properly cited (including links to both the formal publication through the relevant DOI and the license). See: https://creativecommons.org/licenses/by-nc-nd/4.0/.

\section{References}

1. Dupuy DE, Fernando HC, Hillman S, et al. Radiofrequency ablation of stage IA non-small cell lung cancer in medically inoperable patients: Results from the American College of Surgeons Oncology Group Z4033 (Alliance) trial. Cancer 2015;121:3491-8.

2. Garetto I, Busso M, Sardo D, et al. Radiofrequency ablation of thoracic tumours: lessons learned with ablation 
of 100 lesions. Radiol Med 2014;119:33-40.

3. Koelblinger C, Strauss S, Gillams A. Outcome after radiofrequency ablation of sarcoma lung metastases. Cardiovasc Intervent Radiol 2014;37:147-53.

4. Prud'homme C, Deschamps F, Moulin B, et al. Imageguided lung metastasis ablation: a literature review. Int J Hyperthermia 2019;36:37-45.

5. Izzo F, Granata V, Grassi R, et al. Radiofrequency Ablation and Microwave Ablation in Liver Tumors: An Update. Oncologist 2019;24:e990-e1005.

6. Palussi Granata V, Grassi R, et al. Radiofrequency Ablation and Microwaves Ablation for Early Stage Lung Cancer? What Is the Evidence? Curr Oncol Rep 2021;23:81.

7. Yang Q, Qi H, Zhang R, et al. Risk Factors for Local Progression after Percutaneous Radiofrequency Ablation of Lung Tumors: Evaluation Based on a Review of 147 Tumors. J Vasc Interv Radiol 2017;28:481-9.

8. Cheng Z GZ, Shi L, Shan F. The advance of imaging evaluation after CT-guided percutaneous radiofrequency ablation for lung tumors. International Journal of Medical Radiology 2016;39:382-5.

9. Das JP, Barry C, Schhder H, et al. Imaging following thermal ablation of early lung cancers: expected posttreatment findings and tumour recurrence. Clin Radiol 2021;76:864.e13-23.

10. Miles KA, Lee TY, Goh V, et al. Current status and guidelines for the assessment of tumour vascular support with dynamic contrast-enhanced computed tomography. Eur Radiol 2012;22:1430-41.

11. Ippolito D, Bonaffini PA, Capraro C, et al. Viable residual tumor tissue after radiofrequency ablation treatment in hepatocellular carcinoma: evaluation with CT perfusion. Abdom Imaging 2013;38:502-10.

12. Mahnken AH, Klotz E, Schreiber S, et al. Volumetric arterial enhancement fraction predicts tumor recurrence after hepatic radiofrequency ablation of liver metastases: initial results. AJR Am J Roentgenol 2011;196:W573-9.

13. Meijerink MR, van Waesberghe JH, van der Weide L, et al. Early detection of local RFA site recurrence using total liver volume perfusion CT initial experience. Acad Radiol 2009; 16:1215-22.

14. Nakano S, Gibo J, Fukushima Y, et al. Perfusion evaluation of lung cancer: assessment using dual-input perfusion computed tomography. J Thorac Imaging 2013;28:253-62.

15. Milne EN. Circulation of primary and metastatic pulmonary neoplasms. A postmortem microarteriographic study. Am J Roentgenol Radium Ther Nucl Med 1967;100:603-19.

16. Ogilvie RW, Blanding JD Jr, Wood ML, et al. the arterial supply to experimental metastatic VX2 and XY tumors in rabbit lungs. Cancer Res 1964;24:1418-31.

17. Cho YK, Kim Y, Rhim H. Pitfalls in the radiological and pathological correlation of tumour response rates of hepatocellular carcinoma following radiofrequency ablation. J Clin Pathol 2009;62:1071-3.

18. Clasen S, Krober SM, Kosan B, et al. Pathomorphologic evaluation of pulmonary radiofrequency ablation: proof of cell death is characterized by DNA fragmentation and apoptotic bodies. Cancer 2008;113:3121-9.

19. Yan P, Tong AN, Nie XL, et al. Assessment of safety margin after microwave ablation of stage I NSCLC with three-dimensional reconstruction technique using CT imaging. BMC Med Imaging 2021;21:96.

20. Li C, Wang J, Shao JB, et al. Microwave ablation combined with chemotherapy improved progression free survival of IV stage lung adenocarcinoma patients compared with chemotherapy alone. Thorac Cancer 2019;10:1628-35.

21. Yamamoto A, Nakamura K, Matsuoka T, et al. Radiofrequency ablation in a porcine lung model: correlation between CT and histopathologic findings. AJR Am J Roentgenol 2005;185:1299-306.

22. de Baoto A, Nakamura K, Matsuoka T, et al. Radiofrequency ablacacy and survival after radiofrequency ablation of lung tumors with minimum follow-up of 1 year: prospective evaluation. Radiology 2006;240:587-96.

23. Lee JM, Jin GY, Goldberg SN, et al. Percutaneous radiofrequency ablation for inoperable non-small cell lung cancer and metastases: preliminary report. Radiology 2004;230:125-34.

24. Wang L, Che K, Liu Z, et al. Establishment and evaluation of the VX2 orthotopic lung cancer rabbit model: a ultraminimal invasive percutaneous puncture inoculation method. Korean J Physiol Pharmacol 2018;22:291-300.

25. Hamamoto S, Okuma T, Yamamoto A, et al. Radiofrequency ablation and immunostimulant OK432: combination therapy enhances systemic antitumor immunity for treatment of VX2 lung tumors in rabbits. Radiology 2013;267:405-13.

26. Ye X, Fan W; Minimally Invasive and Comprehensive Treatment of Lung Cancer Branch, Professional Committee of Minimally Invasive Treatment of Cancer, Chinese Anti-Cancer Association. Expert consensus for thermal ablation of primary and metastatic lung tumors. Zhongguo Fei Ai Za Zhi 2014;17:294-301.

27. Weidner N. Intratumor microvessel density as a prognostic factor in cancer. Am J Pathol 1995;147:9-19.

28. Pan 1. atlas of experimental pathology techniques. 1 ed. Beijing: China Science Publishing \& Media Ltd., 2014:373-4.

29. Neumann RA, Knobler RM, Pieczkowski F, et al. Enzyme 
histochemical analysis of cell viability after argon laserinduced coagulation necrosis of the skin. J Am Acad Dermatol 1991;25:991-8.

30. Wang Q, Huang J, Ma K, et al. Evaluation of ghost cell survival in the area of radiofrequency ablation. PLoS One 2012;7:e53158.

31. Bressem KK, Vahldiek JL, Erxleben C, et al. Comparison of different 4D CT-Perfusion algorithms to visualize lesions after microwave ablation in an in vivo porcine model. Int J Hyperthermia 2019;36:1098-107.

32. Miles KA. Perfusion CT for the assessment of tumour vascularity: which protocol? Br J Radiol 2003;76 Spec No 1:S36-42.

33. Strauch LS, Eriksen RØ, Sandgaard M, et al. Assessing Tumor Response to Treatment in Patients with Lung Cancer Using Dynamic Contrast-Enhanced CT. Diagnostics (Basel) 2016;6:28.

34. Qiao PG, Zhang HT, Zhou J, et al. Early evaluation of targeted therapy effectiveness in non-small cell lung cancer by dynamic contrast-enhanced CT. Clin Transl Oncol 2016;18:47-57.

35. Tacelli N, Santangelo T, Scherpereel A, et al. Perfusion CT allows prediction of therapy response in non-small cell lung cancer treated with conventional and anti-angiogenic chemotherapy. Eur Radiol 2013;23:2127-36.

36. Fraioli F, Anzidei M, Serra G, et al. Whole-tumour CTperfusion of unresectable lung cancer for the monitoring of anti-angiogenetic chemotherapy effects. Br J Radiol 2013;86:20120174.

37. Fraioli F, Anzidei M, Zaccagna F, et al. Whole-tumor perfusion CT in patients with advanced lung adenocarcinoma treated with conventional and antiangiogenetic chemotherapy: initial experience. Radiology 2011;259:574-82.

38. Hegenscheid K, Behrendt N, Rosenberg C, et al. Assessing early vascular changes and treatment response after laserinduced thermotherapy of pulmonary metastases with perfusion CT: initial experience. AJR Am J Roentgenol 2010;194:1116-23.

39. Parvizi N, Chung D, Little MW, et al. Does perfusion CT play a role in the evaluation of percutaneous microwaveablated lung tumours? Clin Radiol 2016;71:1137-42.

40. Sugaar S, LeVeen HH. A histopathologic study on the effects of radiofrequency thermotherapy on malignant tumors of the lung. Cancer 1979;43:767-83.

41. Yuan X, Zhang J, Quan C, et al. Differentiation of malignant and benign pulmonary nodules with first-pass dual-input perfusion CT. Eur Radiol 2013;23:2469-74.

42. Yuan X, Zhang J, Ao G, et al. Lung cancer perfusion: can we measure pulmonary and bronchial circulation simultaneously? Eur Radiol 2012;22:1665-71.
43. Pontana F, Remy-Jardin M, Duhamel A, et al. Lung perfusion with dual-energy multi-detector row CT: can it help recognize ground glass opacities of vascular origin? Acad Radiol 2010;17:587-94.

44. Primack SL, Müller NL, Mayo JR, et al. Pulmonary parenchymal abnormalities of vascular origin: highresolution CT findings. Radiographics 1994;14:739-46.

45. Abtin FG, Eradat J, Gutierrez AJ, et al. Radiofrequency ablation of lung tumors: imaging features of the postablation zone. Radiographics 2012;32:947-69.

46. Thieme SF, Vahldiek JL, Tummler K, et al. Value or waste: Perfusion imaging following radiofrequency ablation - early experience. Clin Hemorheol Microcirc 2015;61:323-31.

47. Ling S, Deng D, Mo Y, et al. Correlations between CT perfusion parameters and vascular endothelial growth factor expression and microvessel density in implanted VX2 lung tumors. Cell Biochem Biophys 2014;70:629-33.

48. Spira D, Neumeister H, Spira SM, et al. Assessment of tumor vascularity in lung cancer using volume perfusion CT (VPCT) with histopathologic comparison: a further step toward an individualized tumor characterization. J Comput Assist Tomogr 2013;37:15-21.

49. Choi SH, Chung JW, Kim HC, et al. The role of perfusion CT as a follow-up modality after transcatheter arterial chemoembolization: an experimental study in a rabbit model. Invest Radiol 2010;45:427-36.

50. Ma E, Ren A, Gao B, et al. ROI for outlining an entire tumor is a reliable approach for quantification of lung cancer tumor vascular parameters using CT perfusion. Onco Targets Ther 2016;9:2377-84.

51. Herbst RS, Onn A, Sandler A. Angiogenesis and lung cancer: prognostic and therapeutic implications. J Clin Oncol 2005;23:3243-56.

52. Ohno Y, Koyama H, Fujisawa Y, et al. Dynamic contrastenhanced perfusion area detector CT for non-small cell lung cancer patients: Influence of mathematical models on early prediction capabilities for treatment response and recurrence after chemoradiotherapy. Eur J Radiol 2016;85:176-86.

53. Sauter AW, Merkle A, Schulze M, et al. Intraobserver and interobserver agreement of volume perfusion CT (VPCT) measurements in patients with lung lesions. Eur J Radiol 2012;81:2853-9.

Cite this article as: Cheng Z, Wang Y, Yuan M, Liang J, Feng Y, Shi Y, Zhang Z, Shan F. CT perfusion imaging can detect residual lung tumor early after radiofrequency ablation: a preliminary animal study on both tumoral and peri-tumoral region assessment. J Thorac Dis 2022;14(1):64-75. doi: 10.21037/jtd-21-967 


\section{Supplementary}

Table S1 Subgroups with different injection protocol

\begin{tabular}{|c|c|c|c|c|}
\hline \multirow{2}{*}{ Subgroup } & \multicolumn{4}{|c|}{ Injection protocol } \\
\hline & Rate (mL/s) & Volume (mL) & Rate (mL/s) & Volume $(\mathrm{mL})$ \\
\hline 1 & 1.5 & 1.0 & 1.5 & 4.0 \\
\hline 2 & 1.5 & 2.0 & 1.5 & 5.0 \\
\hline 4 & 2.0 & 2.0 & 2.0 & 5.0 \\
\hline
\end{tabular}

The same volume plus additional $3 \mathrm{~mL}$ (the volume of type "Y" connecting tube was $3 \mathrm{~mL}$ ) sodium chloride $(0.9 \%)$ with same rate was injected following injection of iohexol for sake of bolus injection.

Table S2 Intergroup comparisons of key parameters of TDC

\begin{tabular}{|c|c|c|c|c|}
\hline \multirow{2}{*}{ Subgroups } & \multicolumn{4}{|c|}{$P$ value } \\
\hline & $0.0025^{*}$ & $0.0027^{*}$ & $0.0035^{\star}$ & $0.4975^{\star}$ \\
\hline 2 vs. 1 & 0.004151 & 0.000408 & 0.012614 & - \\
\hline 2 vs. 3 & 0.000656 & 0.023337 & 0.000749 & - \\
\hline
\end{tabular}

(I) PA-max-maximum enhancement CT value of pulmonary artery; Tpa-max-time to PA-max; Spa-max-slope of PA-max; AA-max-maximum enhancement CT value of aortic artery; Tpa-aa-time difference to peak of PA-max and AA-max. $P$ value marked with "*' utilizing the standard-a $=0.05$, and the left utilizing the standard-a $=0.004167$. $P$ value in italic indicating significant difference. (II) Intergroup comparisons were made partially, because of the preliminary observation of TDCs getting the targeted protocols. TDC, time-density curve. 

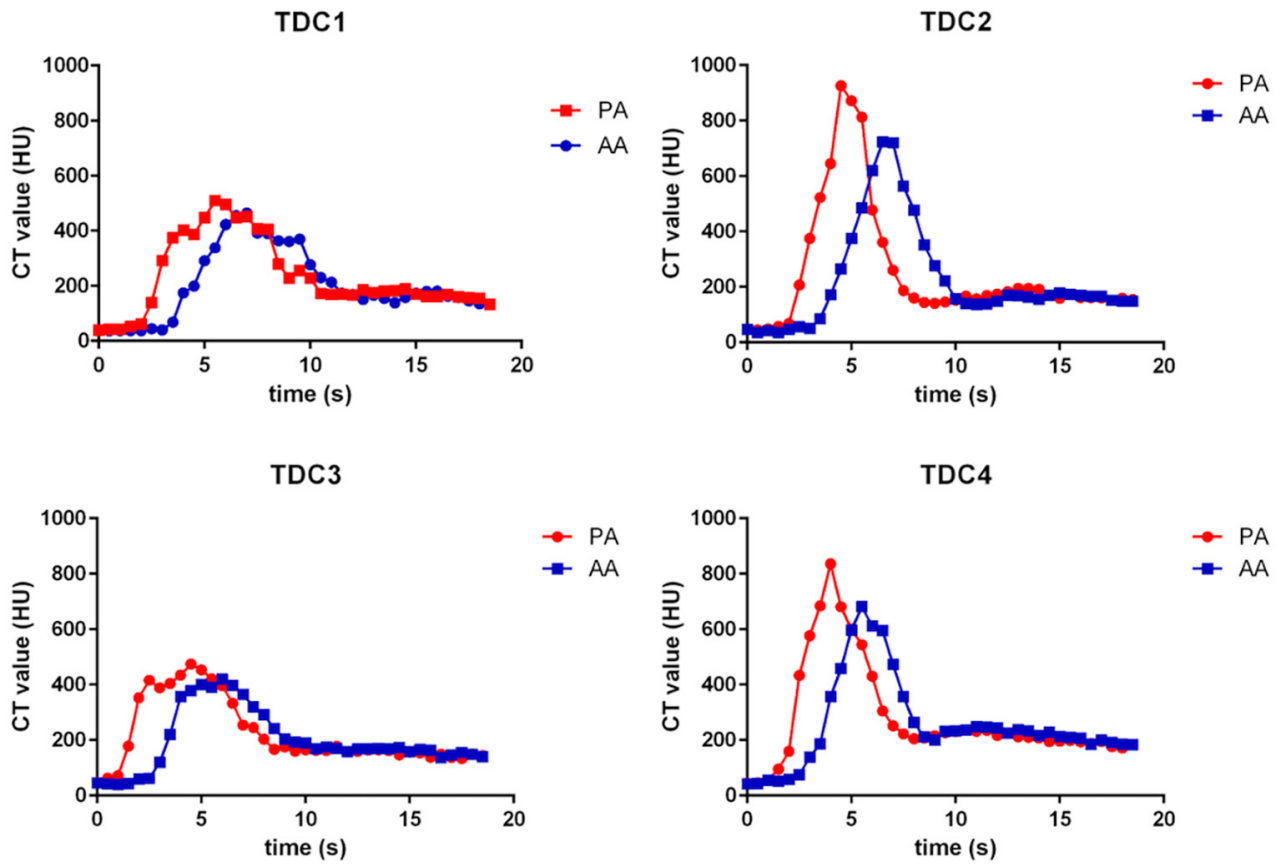

Figure S1 Time-density curves (TDCs) of dual circulation in subgroups. TDCs were similar in an asymmetric bell, and TDCs in the subgroup 2 (TDC2) and the subgroup 4 (TDC4) were sharper than those of subgroup 1 (TDC1) and subgroup 3 (TDC3). 